\title{
La mejora de sistemas de producción de Ingeniería contra pedido: Un estudio empirico
}

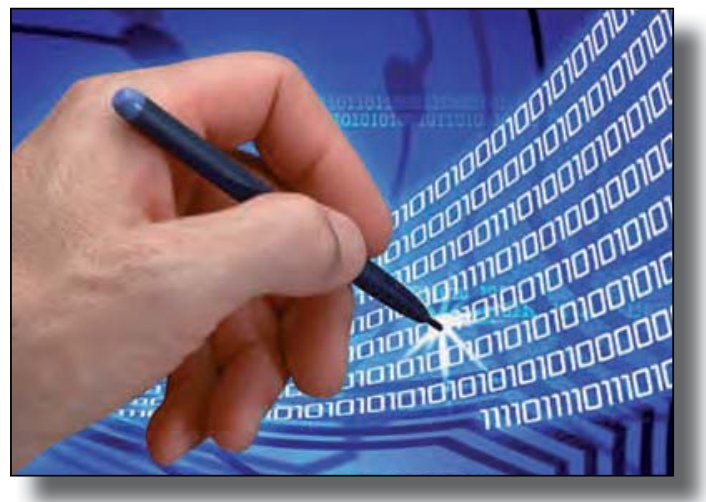

Ander Errasti-Opacua*

Sandra Martínez-Miguélez ${ }^{*}$

Javier Santos-García*

Raúl Poler-Escoto**
Doctor Ingeniero Industrial Ingeniera en Organización Industrial Doctor Ingeniero Industrial Doctor Ingeniero Industrial

* TECNUN. Universidad de Navarra. Paseo Manuel Lardizabal, 13 - 20018 Donostia.

Tfno: +34 943 219877. aerrasti@tecnun.es

** UNIVERSIDAD POLITECNICA DE VALENCIA. Centro de Investigación Gestión e Ingeniería de Producción. Camino de Vera, s/n - 46022 Valencia. Tfno: + 34963879680.

\section{Engineer to Order production systems improvement: An empirical study}

\section{ABSTRACT}

\section{- Introduction}

To investigate the adoption and transfer of some good practices in the Construction Sector is the aim of the following research. The GRAI Methodology and the DGRAl technique are associated to improve the operational performance of the supply chain. They are already known and have been studied by researchers; however the development of these techniques in the field of Construction has not been so deep. Method

The proposed research method is based on Action Research. This is a variant of Case Studies in which the researchers are involved in the changing process. This method has been chosen because it is a theory extension or refinement. In the last three years the researchers have been involved in using an experimental model that uses GRAl and DGRAI for the diagnosis, strategy formulation and deploy of these practices to improve the performance of one top firms related to Construction sector.

Results

Two years after the starting up of the reengineering process has improved the Quality of Service and reduced fixed costs due to the reduction of stocks needed.

Conclusion

It has shown that the benefits of subcontractors and suppliers integration, the improvement of planning and control system of the extended enterprise aided by GRAl and DGRAl as well as Action Research utility for this type of Research are completely demonstrated. - Keywords: Project Management, GRAI DGRAI, Engineer to Order, Construction sector and Mass Customization.

\section{RESUMEN}

\section{Introducción}

El objetivo del siguiente estudio es investigar la adopción y transferencia de algunas buenas prácticas al Sector de la Construcción. La Metodología GRAI y la técnica DGRAI están asociadas a la mejora del rendimiento operativo de la cadena de suministro siendo ya conocidas y estudiadas por investigadores. Sin embargo, su desarrollo en el ámbito de la construcción no ha sido tan profundo.

\section{Método}

El método de investigación propuesto se basa en la Experimentación Activa o Action Research. Este método es una variante de los Estudios de Caso en la cual los investigadores están involucrados en el proceso de cambio. En los últimos tres años, los investigadores han estado implicados en el uso de un modelo experimental que utiliza GRAI y DGRAI para el diagnóstico, la formulación y el despliegue de la estrategia de operaciones para mejorar el desarrollo de una empresa relacionada con el Sector de la Construcción.

\section{Resultados}

Después de dos años desde el comienzo del proceso de reingeniería se ha mejorado la Calidad de Servicio y se han reducido los costes de inmovilizado debido a la reducción de los stocks necesarios.

\section{Conclusiones}

Se ha demostrado los beneficios de la integración de subcontratistas y proveedores, de la mejora del sistema de planificación, del control de la producción y del aprovisionamiento de la empresa con la ayuda de GRAI y DGRAI, así como la utilidad de la Action Research para este tipo de investigación. 
Palabras clave: Producción por Proyectos, GRAI, DGRAI, Ingeniería Bajo Pedido, Personalización en Masa, Sector de la Construcción.

\section{INTRODUCCIÓN Y REVISIÓN DE LA LITERATURA}

\section{1. INTRODUCCIÓN}

La posibilidad de transferir buenas prácticas de producción al Sector de la Construcción han sido identificadas en la literatura (Ngowi, 2000) (Anumba et al, 2000), en particular Ngowi (2000) ha sugerido que: "La gestión de la cadena de suministro y las técnicas de reducción del plazo de suministro requieren de una estrecha cooperación interfuncional para ser implantadas con éxito en los sistemas productivos y existe la posibilidad de su utilización en el sector de construcción a nivel de proyecto". Además, se ha argumentado que la implementación de estos principios pueden ser extendidos más allá de los límites de las organizaciones llegando a incluir a sus proveedores (Gunasekaran, 1998) (Towill, 1999). De hecho, un número cada vez mayor de publicaciones se refieren a proyectos entre empresas (Soderlund, 2004).

Aunque existen evidencias de la aplicación de estos principios en empresas fabricantes y subcontratistas principales en el Sector de Construcción (Errasti et al, 2006) (Errasti et al 2008), hay pocas evidencias de su desarrollo en entornos que realizan su producción, una vez se haya realizado la ingeniería según el pedido del cliente y más concretamente en el Sector de la Construcción.

Este artículo se estructura del siguiente modo: se comienza con una revisión de la literatura sobre las Cadenas de Suministro en el Sector de la Construcción que trabaja por proyectos y de los principios asociados, de la Personalización en Masa y de la integración de los proveedores. Seguidamente se presenta la pregunta de investigación y se expone el método que los investigadores han llevado a cabo para alcanzar los Objetivos de la Investigación, que incluyen la construcción de la teoría y el testeo de la teoría en una empresa real. Por último, se exponen los resultados alcanzados en la experimentación, las conclusiones y las futuras líneas de investigación.

\section{REVISIÓN DE LA LITERATURA}

\section{1. CADENAS DE SUMINISTRO EN EL SECTOR DE LA CONSTRUCCIÓN}

En el Sector de la Construcción, los diseñadores definen el producto cumpliendo con los requisitos del cliente, tanto de diseño como de costes. Posteriormente, se entrega el proyecto al contratista que será el responsable de la construcción total de la instalación o infraestructura. Suele ser habitual el hecho de que el contratista subcontrate parte del trabajo que tiene a su cargo, eligiendo a subcontratistas especializados en los distintos conjuntos a suministrar.
Los proyectos de construcción, por lo general, requieren de la contribución de proveedores con conocimientos especializados abarcando una amplia gama de disciplinas tanto de la construcción como de la producción (Fig.1).

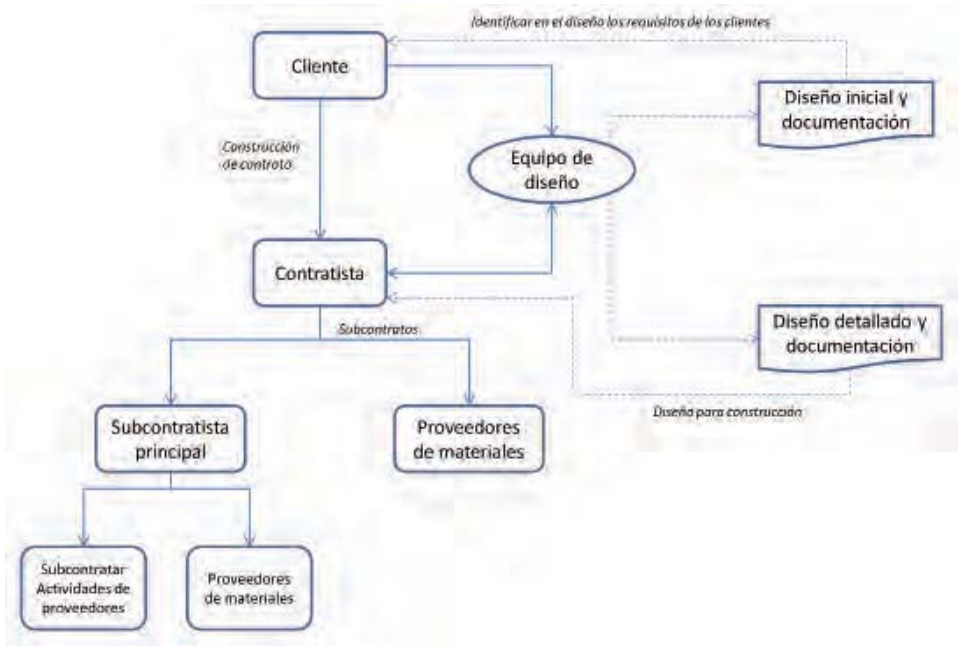

Fig. 1: Agentes involucrados en el diseño y la construcción de proyectos y sus relaciones, adaptada de Thomas (2002)

Estos subcontratistas están sujetos a una gran presión en términos de calidad, servicio y coste (Chan et al, 1999). Con frecuencia aparecen conflictos entre los acuerdos contractuales y el resultado real en plazo, calidad y coste del suministro, lo cual se trata de atenuar mediante el establecimiento de sanciones y penalizaciones por no cumplimiento (Haque, 2003).

Los subcontratistas están, además, obligados a crear valor si quieren sobrevivir en un ambiente competitivo, que cada vez es más global en su alcance y dependiente en el desarrollo de la red de proveedores. El valor en este contexto está definido como "la cantidad que el cliente está dispuesto a pagar por lo que una compañía es capaz de ofrecerles" (Porter, 1980). Debido a esto, algunos autores (Porter, 1985) han sostenido que el análisis de valor no debería estar únicamente restringido al valor añadido por la compañía, sino que se debería incluir al resto de agentes de la cadena de suministro, por ejemplo, los proveedores, el diseño de los equipos, los contratistas, los subcontratistas y resto de agentes involucrados. En consecuencia, el concepto de Cadena de Valor (Porter, 1985) debe extenderse y abarcar todos los agentes de la red de distribución que contribuyen a la propuesta de valor. En este sentido, el desarrollo de una estrategia de cadena de suministro que proporcione una perspectiva holística de la Cadena de Valor es muy importante ya que se asegurará que las estrategias de los distintos agentes están alineadas con los objetivos generales del proyecto.

En este contexto, los subcontratistas deben desarrollar estrategias de producción que tengan en cuenta la posición de la compañía en la cadena de valor (Thomas et al, 
2002). En el Sector de Construcción, los contratistas y subcontratistas trabajan dentro de un sistema basado en proyectos ("Engineer to Order") y la tendencia en dicha gestión es hacia una mayor integración de la organización (Schemerhorn, 1993). Las oportunidades de repetición de producciones son, por lo general, limitadas, en otras palabras se trata de un entorno de "bajo volumen y alta variedad" (Slack, 2004) donde la mano de obra, el equipamiento y los materiales deben ser trasladados al lugar donde el producto se monta final. Además, los proyectos de construcción se caracterizan frecuentemente por un alto grado de incertidumbre operativa (Kolveit, 2004). Pueden transcurrir semanas o incluso meses para completar el montaje de un gran número de componentes y subsistemas que se realizan fuera de sitio.

2.1.1. Principios de la personalización en masa: paradigma de los sistemas de producción por proyectos

Haque (2003) afirma que las razones para poner en marcha un programa de mejora de una empresa subcontratista son: mejorar la calidad del producto, reducir el tiempo de mercado, reducir los costes de producción y mejorar la eficiencia de los recursos humanos. El tiempo de suministro al cliente, se entiende cada vez más como una fuente importante de ventaja competitiva (Mahmoud-Jouini et al, 2004) también en el sector de construcción (Errasti et al, 2006). Otro elemento diferenciador importante es cómo los clientes valoran el nivel de personalización y adaptación de los productos a sus necesidades. La creación de productos únicos sólo es posible si el cliente puede influir sobre los requerimientos, es decir, el producto tiene que estar por definir cuando el cliente expresa sus necesidades y, una vez realizado el pedido, será necesario realizar un proceso de ingeniería de detalle según las especificaciones que el cliente solicita. A esta forma de suministro se le denomina ingeniería contra pedido o "Engineer to Order "(ETO). En este contexto, las actividades de diseño, compra y aprovisionamiento, fabricación y montaje se efectúan mientras el cliente está esperando, pero, algunas de las actividades puede que tengan que desarrollarse basadas en previsiones debido al hecho de que el tiempo de todo el proceso o periodo de maduración es mayor que el tiempo de entrega requerido por el cliente.

Un concepto de uso frecuente para captar este aspecto de la estrategia de operaciones es el punto de desacople (DP, Decoupling Point), entendiéndose como el punto que indica el grado de penetración del pedido del cliente en el flujo de materiales (de la cadena logística) (Hoekstra y Romme, 1992). Este punto separa las actividades dirigidas por los pedidos de los clientes (aguas abajo), de las actividades dirigidas por previsión y planificación (aguas arriba). Típicamente, mediante el posicionamiento del DP, se definen cuatro tipos de fabricación: fabricación contra inventario o stock (Make to Stock), fabricación contra pedido (Make to Order), montaje bajo pedido (Assemble to Order) e ingeniería contra pedido (Engineer to Order) (Olhager, 2003). En el sector de la construcción es habitual el desarrollo de proyectos según ingeniería bajo pedido. Eso significa que el proyecto se construirá bajo pedido, lo que significa que las actividades de valor añadido de fabricación, incluso las de montaje, son desencadenadas por pedidos en lugar de por pronósticos. Mediante la realización de actividades de valor añadido bajo pedido, una compañía evitaría incurrir en los riesgos de previsiones de eventos inciertos (Salvador et al, 2005). Rudberg (2004) introduce la dimensión de la ingeniería en el punto de desacoplamiento del pedido del cliente (Customer Order Decoupling Point, CODP). Una vez que el producto está diseñado se podría interpretar como si el diseño del producto está ya en stock o en stock virtual listo para ser fabricado y montado cuando sea necesario.

Estos sistemas de producción, llamados ingeniería contra pedido, normalmente son sistemas de producción por proyectos donde los elementos de repetición son limitados, es decir, "de bajo volumen y alta variedad" (Slack et al, 2004)

Sin embargo, la Producción en Masa Personalizada podría permitir aumentar el volumen de producción. En este caso, se tendrían sistemas de producción por proyectos de "alto volumen y alta variedad". Por lo tanto, la aplicación de este concepto ayudaría a las empresas a acortar ambos tiempos (de producción y entrega) y ofrecer valor a los clientes en términos de productos únicos dentro de un sistema de alto volumen de producción.

\subsubsection{Integración de los proveedores en la industria} de la construcción

En el Sector de la Construcción, ningún proyecto puede ser realizado por una sola organización sin un cierto grado de subcontratación, incluso de diseño o método de construcción (Anumba et al, 2000). Rara vez se puede decir que una sola empresa posee toda la experiencia técnica, recursos o capital de inversión necesario. "Los contratistas principales y los proveedores deben ser introducidos en la fase de diseño para que las actividades aguas abajo del proceso de construcción puedan ser abordadas" (Gunasekaran, 1998).

Los proveedores, por lo general, se dedican a la fabricación de componentes o subsistemas que posteriormente suministrarán para que se proceda al ensamblaje final en los lugares de fabricación o montaje. Algunos contratistas puede que sean expertos en algunos de los componentes o subsistemas, por lo que suministran en más de un proyecto.

El diseño de detalle de los componentes no es secuencial con el aprovisionamiento y la fabricación. Esto crea procesos múltiples y paralelos. Las operaciones en paralelo son al mismo tiempo interdependientes, por lo que generan interrupciones y perturbaciones en los procesos de fabricación y montaje.

Los principales fabricantes subcontratistas responden a estos desafíos trabajando más estrechamente con sus proveedores y clientes a través de la creación de una empresa que se extiende en toda la cadena de valor. Eso significa la vinculación en términos de coordinación en el diseño, el desarrollo y el coste de empresas independientes (Jagdev et 
al, 1998) y una estrecha relación y compromiso entre los fabricantes y proveedores en función de un intercambio eficaz de información para tener un mejor rendimiento (Jagdev et al, 1998) (Neng et al, 1995) (Childe, 1998) (Yu et al, 2001).

Aún cuando muchas empresas están sacrificando la rentabilidad y la satisfacción del cliente, porque no son capaces de colaborar con los proveedores, no todas las relaciones comerciales son colaborativas (Spekman, 1998). Los compradores son mucho más escépticos sobre los beneficios que ofrece una integración, porque son conscientes de la dependencia que genera este tipo de forma de trabajo, debido a que fuerza a trabajar con un número más reducido de proveedores.

Las ventajas que se podrían obtener en el Sector de Construcción gracias a una colaboración estrecha son:

- Coste y ahorros de la calidad en el proyecto durante el diseño del proceso (Thomas, 2002)

- La ejecución del proyecto podría ser más eficiente si la coordinación operativa evita malentendidos en el proceso de fabricación.

- La ejecución del proyecto podría ser más eficiente si el proyecto de fabricación y la facilidad de montaje se tienen en cuenta (Anumba, 2000).

La falta de aplicación de principios de cadena de suministro y gestión de la logística en un entorno de proyectos tiene una influencia significativa en los sistemas de fabricación. Además, a esto hay que añadir que el menor desempeño en costes y plazos puede ser atribuible a fallos de calidad, malos entendidos y falta de comunicación entre los participantes.

Es evidente que, para aprovechar las ventajas y controlar los riesgos, las relaciones de colaboración entre empresas en el Sector de Construcción tienen gran potencial. Esto significa que las compañías deben rediseñar sus procesos de negocio operativos para facilitar mejoras en el intercambio de información, el desarrollo de estrechas relaciones de cooperación y en última instancia, la colaboración en proyectos de beneficio mutuo.

\subsubsection{Metodología GRAI}

El modelado empresarial es una herramienta que ofrece un adecuado soporte para afrontar este tipo de problemas. Los métodos de modelado ayudan a realizar la reingeniería de procesos asociados a una empresa o una cadena de suministro. En las últimas décadas han emergido varias arquitecturas para el análisis y rediseño de sistemas empresariales, entre ellas destacan: CIMOSA, GIM y PERA.

Uno de los subsistemas más complejos de análisis y diseño de una organización es el sistema de decisiones. La arquitectura GIM y, en particular, la metodología GRAI (Doumeingts, 1984) permite el estudio de los Sistemas de Gestión de Producción (SGP) desde el punto de vista de las decisiones que se toman, por tanto, tiene por objeto dar soporte al análisis y rediseño de sistemas de decisión. Se centra en el análisis del conjunto de decisiones que se toman a nivel del SGP tanto en los modos normales de funcionamiento como en los perturbados.

GIM parte de la construcción del modelo conceptual del sistema actual (etapa de análisis) y evoluciona hacia su conversión al modelo conceptual del futuro sistema (etapa de diseño). Por tanto, se centra en la etapa de análisis y diseño del sistema decisional y lo analiza a nivel conceptual y estructural

A nivel conceptual, el modelo GRAI está compuesto de tres sistemas: el sistema físico, el sistema de decisión y el sistema de información (Fig.2).

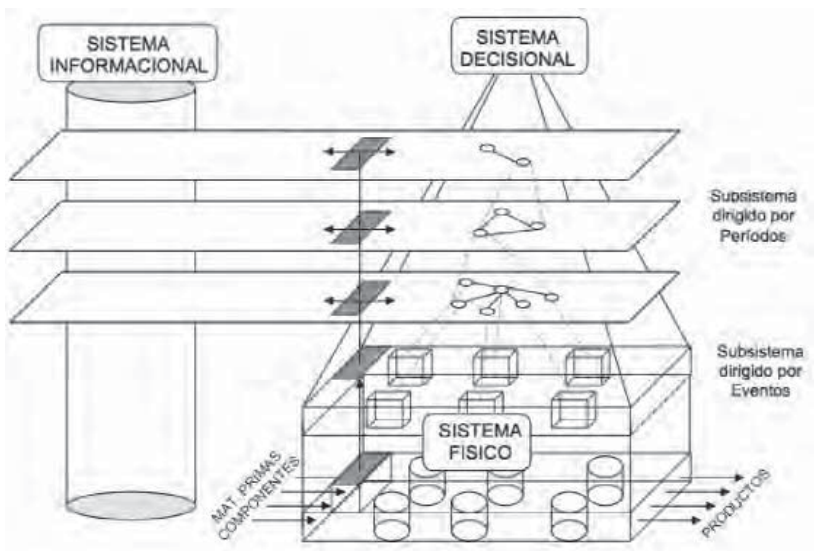

Fig.2: Sistemas del modelo GRAI

El sistema de decisión está dividido en niveles de decisión. Por lo tanto, GRAI propone una estructura jerárquica para definir el sistema de decisión de una empresa. El formalismo de modelado utilizado para la representación de los centros de decisión es la Rejilla GRAI. Dichos centros de decisión se organizan en la rejilla según funciones y niveles temporales de decisión (Fig.3).

\begin{tabular}{|l|l|l|l|l|l|l|}
\hline & $\begin{array}{c}\text { Function } \\
1\end{array}$ & $\begin{array}{c}\text { Function } \\
2\end{array}$ & $\begin{array}{c}\text { Function } \\
3\end{array}$ & $\ldots$ & $\begin{array}{c}\text { Function } \\
n-1\end{array}$ & $\begin{array}{c}\text { Function } \\
n\end{array}$ \\
\hline $\begin{array}{l}\mathrm{H} 1 \\
\mathrm{P} 1\end{array}$ & & & & & & \\
\hline $\mathrm{H} 2$ \\
$\mathrm{P2}$
\end{tabular}

Fig.3: Rejilla GRAl

Para el análisis y mejora de sistemas de decisión se parte de modelos de referencia, en los cuales se estructuran las funciones, niveles y centros de decisión que deberían existir en cada tipo de sistema. La Fig. 4 muestra un modelo de referencia para un Sistema de Gestión de Producción. 


\begin{tabular}{|c|c|c|c|c|c|c|c|}
\hline & $\begin{array}{c}\text { Informaciones } \\
\text { Externas }\end{array}$ & $\begin{array}{l}\text { Gestionar } \\
\text { los } \\
\text { Productos } \\
\text { COMPRAS }\end{array}$ & $\begin{array}{l}\text { Gestionar los } \\
\text { Productos } \\
\text { APROVISIón }\end{array}$ & PLANIFICAR & $\begin{array}{l}\text { Gestionar los } \\
\text { Recursos } \\
\text { TÉCNICOS }\end{array}$ & $\begin{array}{l}\text { Gestionar los } \\
\text { Recursos } \\
\text { HUMANOS }\end{array}$ & $\begin{array}{l}\text { Informaciones } \\
\text { Internas }\end{array}$ \\
\hline $\begin{array}{c}\mathrm{H}=1 \text { año } \\
\mathrm{P}=6 \text { meses }\end{array}$ & $\begin{array}{c}\text { Información del } \\
\text { entorno, } \\
\text { mercado, } \\
\text { competencia }\end{array}$ & $\begin{array}{c}\text { Plan Gestión } \\
\text { Compras. } \\
\text { Negociar } \\
\text { contratos, } \\
\text { fijar } \\
\text { parámetros } \\
\text { de compra. } \\
\text { Búsqueda de } \\
\text { proveedores } \\
\end{array}$ & $\begin{array}{c}\text { Plan Gestión } \\
\text { Aprovisionamiento }\end{array}$ & $\begin{array}{l}\text { Plan Gestión } \\
\text { Producción }\end{array}$ & Inversión a LP & 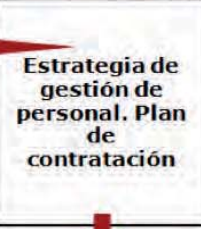 & \\
\hline $\begin{array}{c}\mathrm{H}=6 \text { meses } \\
\mathrm{P}=1 \text { mes }\end{array}$ & $\begin{array}{l}\text { Ofertas. } \\
\text { Previsiones de } \\
\text { demandas }\end{array}$ & $\begin{array}{l}\text { Comprar } \\
\text { artículos } \\
\text { críticos }\end{array}$ & 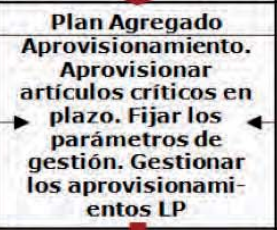 & $\rightarrow$ & $\begin{array}{c}\text { Adaptación de } \\
\text { los recursos. } \\
\rightarrow \begin{array}{c}\text { Reglas de } \\
\text { subcontra- } \\
\text { tación }\end{array}\end{array}$ & $\begin{array}{l}\text { Programas de } \\
\text { formación. } \\
\text { Adaptación de } \\
\text { efectivos }\end{array}$ & $\begin{array}{c}\text { Nivel de stock } \\
\text { de artículos } \\
\text { críticos. Cargas } \\
\text { agregadas }\end{array}$ \\
\hline \multirow[t]{2}{*}{$\begin{array}{c}\mathrm{H}=1 \text { mes } \\
\mathrm{P}=1 \text { semana }\end{array}$} & \multirow[t]{2}{*}{ Pedidos } & $\begin{array}{c}\text { Programa de } \\
\text { compra } \\
\text { artículos no } \\
\text { crísticos }\end{array}$ & $\begin{array}{c}\text { Aprovisionar } \\
\text { artículos según } \\
\text { MRP, SRC y SRP }\end{array}$ & \multirow[t]{2}{*}{$\begin{array}{c}\text { Plan Maestro } \\
\text { Producción. } \\
\text { Planificación } \\
\rightarrow \text { de las cargas } \\
\text { de producción } \\
\text { y necesidades } \\
\end{array}$} & $\rightarrow \begin{array}{c}\text { Reserva de } \\
\text { capacidad }\end{array}$ & $\begin{array}{l}\text { Ajustar los } \\
\text { efectivos }\end{array}$ & \multirow[t]{2}{*}{$\begin{array}{c}\text { Nivel de stock. } \\
\text { Cargas }\end{array}$} \\
\hline & & & & & & & \\
\hline Tiempo Real & $\begin{array}{l}\text { Pedidos } \\
\text { urgentes }\end{array}$ & $\begin{array}{c}\text { Seguimiento } \\
\text { de } \\
\text { incidencias y } \\
\text { acciones de } \\
\text { mejora } \\
\text { definitivas }\end{array}$ & $\begin{array}{c}\text { Programáación } \\
\text { entradas y } \\
\text { suministro a línea } \\
\text { de producción. } \\
\text { Reprogramación des } \\
\text { aprovisionamiento, } \\
\text { gestión de } \\
\text { incidencias y } \\
\text { acciones correctoras } \\
\end{array}$ & $\begin{array}{l}\text { Programación } \\
\text { de fabricación } \\
\text { y gestión } \\
\text { incidencias }\end{array}$ & $\rightarrow \begin{array}{c}\text { Asignar } \\
\text { recursos }\end{array}$ & $\begin{array}{l}\text { Asignar } \\
\text { personal }\end{array}$ & $\begin{array}{c}\text { En curso. } \\
\text { Avance. Stocks }\end{array}$ \\
\hline
\end{tabular}

Fig. 4: Modelo de referencia de un SGP

El modelo DGRAI (Poler et al., 2002) es una extensión del método GRAI, cuyo objetivo básico es la simulación de sistemas de decisión. DGRAI permite simular los procesos de decisión a capacidad finita de los recursos humanos involucrados en tareas de decisión (Poler and Lario, 2001). La simulación permite identificar los problemas asociados a la coordinación de las decisiones, los cuellos de botella en los agentes que deciden y el impacto de los retardos en la ejecución de actividades de toma de decisión. Aunque existen otras propuestas relacionadas con la simulación de sistemas de decisión en el marco del método GRAI (Akif, 1991) (Wadhwa, 1998), solo proponen una simulación macro, a nivel de rejilla GRAI, mientras que DGRAI permite simular al nivel de actividades de toma de decisión. Por otro lado, el modelo DGRAI permite realizar un modelado más natural de un sistema de toma de decisiones periódicas y multinivel (estratégico, táctico y operativo) respecto a otros modelos más apropiado para el análisis de procesos de negocio (Aguilar-Sommar y Poler, 2006). DGRAI permite el modelado de sistemas de decisión de varias empresas pertenecientes a una cadena de suministro y ha sido aplicado a diferentes sectores, como el de automoción (Hernández et al., 2008). En el presente estudio se extiende el modelo DGRAI a cadenas de suministro con sistemas de producción de ingeniería bajo pedido con la finalidad de analizar el impacto de la localización de los proveedores y los períodos de tránsito.

\section{OBJETIVOS}

Aunque algunos autores sostienen que en realidad son pocas las empresas dedicadas a poner en marcha un proceso de integración de agentes (clientes, fabricantes y proveedores) en una cadena de suministro (Fawcett et al, 2002), los autores de este artículo establecen que la metodología GRAI es de gran utilidad para afrontar un proyecto de reingeniería con el fin de mejorar el rendimiento de la cadena de suministro global. Para la mejora de un sistema de producción de ingeniería contra pedido (ETO) se requiere un nuevo enfoque de planificación y control de producción y aprovisionamiento y de evaluación de las necesidades de coordinación entre agentes que permita tener entregas fiables con menores plazos de entrega. El modelo DGRAI permite la simulación de los sistemas de decisión a fin de validar los sistemas de planificación y control de producción propuestos. Estas herramientas se han aplicado ya con éxito en sistemas de producción contra stock (Errasti et al, 2006) y parcialmente en sistemas de producción de ingeniería bajo pedido (Errasti et al, 2007). Sin embargo, la necesidad de la coordinación y sincronización no ha sido estudiada en profundidad en estudios de caso de "alto volumen-alta variedad".

Por lo tanto, este artículo explora el método en el cual el rediseño de los procesos de planificación y control de producción de aprovisionamiento externos e internos deberían integrarse; con la ayuda de la rejilla GRAI y el modelo DGRAI, con el objetivo de mejorar el rendimiento 
global de una cadena de suministro que produce bajo pedido una vez realizada la ingeniería.

\section{MÉTODO}

La metodología de investigación del trabajo que se muestra en este artículo es:

- Construcción de la teoría: se comienza con una extensa revisión bibliográfica para identificar los factores a considerar en la aplicación de la metodología GRAI y la técnica DGRAI a las cadenas de suministro.

- Testeo de la teoría: esta fase ha sido diseñada alrededor de los principios del Action Research que puede verse como una variante de las investigaciones del estudio de caso (Yin, 1994), en los cuales el investigador no es un observador independiente (Westbrook, 1995), (Vignalli, 2003)...

Esta investigación se ha validado aplicándola a un caso real para dar respuesta a las dificultades mencionadas anteriormente.

\subsection{MÉTODO CONSTRUCCIÓN DE LA TEORÍA: FACTORES A CONSIDERAR AL IMPLEMENTAR GRAI EN UNA CADENA DE SUMINISTRO}

El rediseño de un sistema de decisiones en una cadena de suministro supone importantes implicaciones en todas las áreas de la red productiva y logística (Acur, 2000). Por lo tanto, la decisión de acometer un proyecto de este tipo debe calificarse de estratégica.

Para el desarrollo de la estrategia, son necesarias cinco etapas (entradas, análisis, formulación de la estrategia, implementación de la estrategia y revisión de la misma) y para este propósito se pueden utilizar herramientas analíticas y de gestión.

Los autores de este artículo han aceptado este acercamiento, sin embargo esta investigación simplifica este proceso y lo adapta a la estrategia de operaciones de la unidad de negocio (Platts, 1990) considerando los siguientes factores. La metodología o guía tiene en cuenta la posición de la unidad de negocio en la Cadena de Valor (Browne, 1995) y establece las etapas que ayudarán a crear valor (Porter, 1980). En esta etapa, la rejilla GRAI y modelo DGRAI (Poler et al, 2002) respaldan el análisis del sistema actual y del sistema futuro. El diagnóstico, por un lado, contribuye a elegir el contenido de la estrategia (Gunn, 1987) y definir o formular la estrategia (Platts, 1990). Por otro, ayuda a monitorizar las ventajas y desventajas de los futuros sistemas de decisión relacionados con la tecnología de información (especificaciones de hardware y software), tecnología de fabricación (especificaciones de herramientas y equipos) y organización (sistema físico y estructura de gestión).

Posteriormente, se establece una etapa de despliegue de la estrategia formulada (Feurer, 1995). El despliegue de la estrategia es una tarea orientada a los proyectos (Marucheck et al, 1990), donde se establece un proceso de monitorización y revisión de la estrategia para facilitar la alineación de la organización a dicha estrategia. En la Figura 5 se ilustra el método descrito.
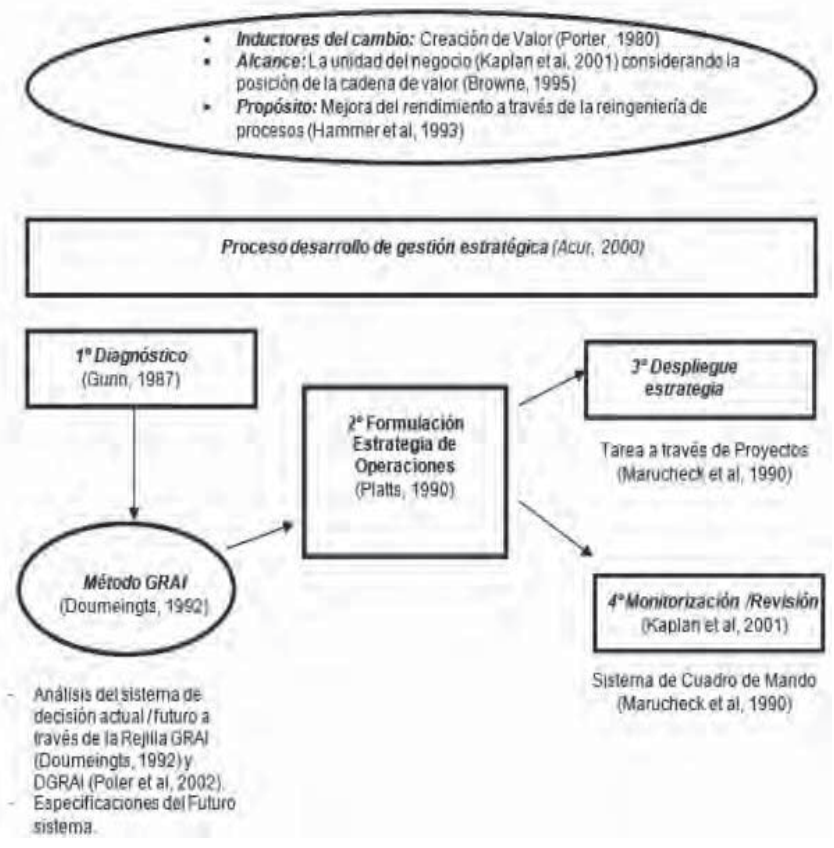

Fig. 5: Representación esquemática del método y factores a considerar (Errasti et al, 2006)

\subsection{TESTEO DE LA TEORÍA: ESTUDIO EMPÍRICO}

El fabricante principal que ha liderado la mejora de la cadena de suministro, pertenece a un grupo industrial español dedicado al diseño, fabricación y montaje de ascensores. Con el objetivo de facilitar la comprensión del estudio de caso, las principales características de la cadena de suministro externa (distribuidores y red de proveedores) y la cadena de suministro interna (almacén de componentes y planta de producción) se describen en esta sección (Fig. 6).

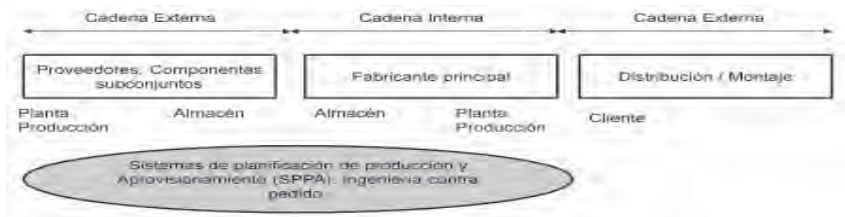

Fig.6: Cadena de suministro interna y externa considerada en el estudio de caso

En la cadena de suministro externa de distribuidores los módulos y los subsistemas de los ascensores son entregados por los distribuidores al lugar de la construcción, donde son montados.

En la cadena de suministro interna, el principal subcontratista planifica su producción cuando el equipo de diseño termina la etapa de personalización de cada ascensor o escalera mecánica. Es ahí cuando la planta de producción trabaja como un sistema de producción de ingeniería 
bajo pedido. Esta planta está basada en los conceptos de producción en masa personalizada, por lo que intenta explotar las ventajas de la producción en masa junto con la personalización del producto para cada cliente. Para este propósito el producto debe tener alta modularidad y partes comunes que permitan una alta personalización, combinando diferentes módulos en la parte final del proceso (montaje).

En la cadena de suministro externa de proveedores, teniendo en cuenta el volumen de logística o el peso por unidad suministrada, número de referencias, distancia de los proveedores y valor por unidad suministrada, los proveedores se clasifican como proveedores J.I.T. volumen, proveedores J.I.T. subconjuntos, proveedores tradicionales contra pedido y proveedores tradicionales contra stock. (Fig. 7)

\begin{tabular}{|c|c|c|c|c|}
\hline retrots & 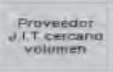 & 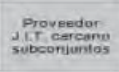 & 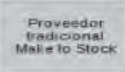 & $\begin{array}{l}\text { Praveedor } \\
\text { sajuco ol } \\
\text { make to ordat }\end{array}$ \\
\hline 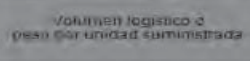 & $\uparrow \uparrow \uparrow$ & $\uparrow \uparrow$ & $\uparrow$ & $\uparrow \uparrow$ \\
\hline 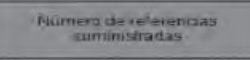 & $\uparrow$ & $\uparrow \uparrow \uparrow$ & $\uparrow$ & $\uparrow \uparrow$ \\
\hline 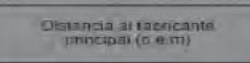 & $\uparrow$ & $\uparrow$ & $\uparrow \uparrow \uparrow$ & $\uparrow \uparrow$ \\
\hline 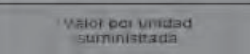 & $\uparrow$ & $\uparrow \uparrow \uparrow$ & $\uparrow \uparrow$ & $\uparrow \uparrow$ \\
\hline
\end{tabular}

Fig.7: Clasificación de proveedores teniendo en cuenta los factores logísticos

El fabricante principal, cuya cadena de suministro es motivo de análisis, pretende mejorar la eficiencia de su cadena de suministro para obtener, de este modo, una ventaja competitiva sostenible. Para este propósito, el equipo investigador ha facilitado esta labor asistido por el método desarrollado (Fig.5). En la fase diagnóstico para el análisis del sistema actual y el diseño del sistema futuro se ha utilizado la rejilla GRAI (Fig.8)y la simulación mediante DGRAI. En la Fig. 8 se pueden apreciar todos los centros de decisiones existentes en la empresa y las relaciones que existen entre ellos.

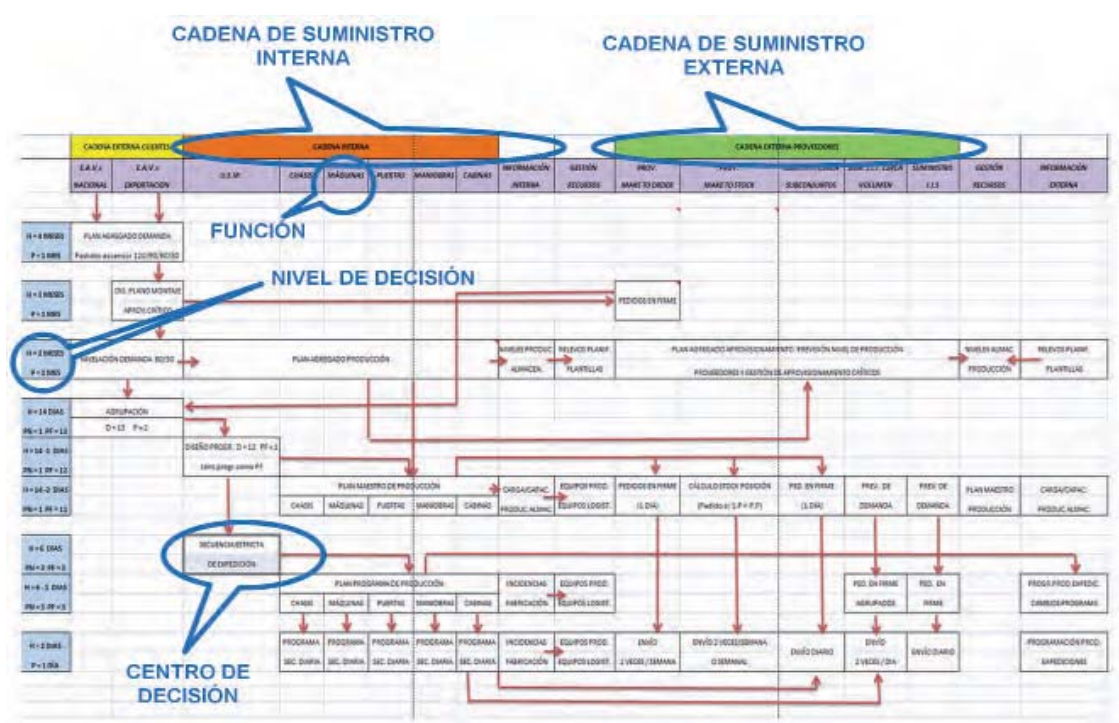

Fig.8: Rejilla GRAl aplicada a la empresa
Los objetivos concretos perseguidos fueron la mejora de la calidad de servicio (reducir el plazo de entrega y cumplimentar en fecha el mayor porcentaje los pedidos realizados) a través del rediseño del sistema de planificación de producción y aprovisionamiento (SPPA) del fabricante y la red de proveedores, así como reducir el coste total de la cadena de suministro. Para explicar la metodología seguida basándose propiamente en el caso de dicha empresa, se puede observar la Fig. 9.
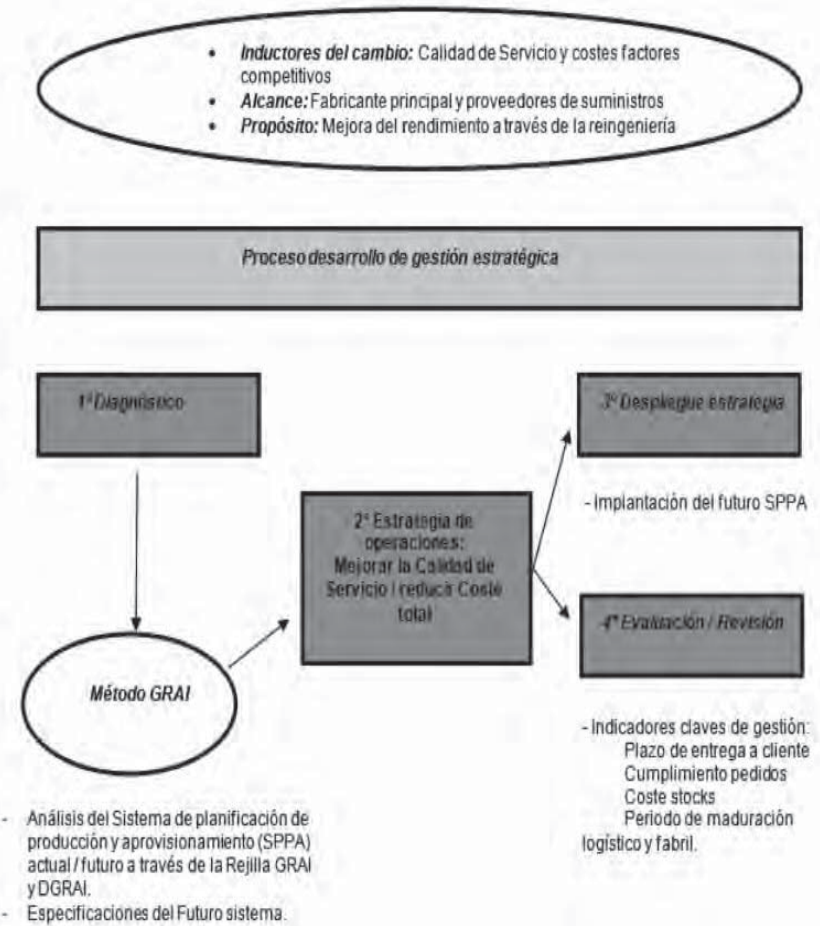

Fig.9: Representación esquemática de la metodología adaptada al caso

\section{RESULTADOS}

\subsection{ANÁLISIS DEL SISTEMA ACTUAL DE LA CADENA DE SUMINISTRO (AS IS) Y DISEÑO DEL FUTURO SISTEMA (TO BE)}

Para analizar la producción y el sistema de planificación de inventario y material (SPIM) actual de la cadena de suministro interna y externa $(A s I s)$, se monitoriza el sistema de decisión empleando la rejilla GRAI, mostrando las principales características del sistema de decisión (niveles de decisión, centros de decisión, periodos de planificación, frecuencias de planificación, alternativas de decisión, información, etc.).

El SPIM debe ser diseñado para equilibrar la oferta y la demanda, manteniendo el flujo de materiales y el valor añadido de la actividad en la fabricación sin 
interrupciones. Se trabaja bajo pedido una vez que la etapa de ingeniería está realizada. El horizonte de planificación es de 20 días y el periodo de planificación es de 2 días. A esta combinación se le denomina " $\mathrm{D}+20: 2$ ", ya que las plantas productivas suministran a los distribuidores los ascensores para montar, que ha acabado su diseño en el día "D", 20 días después. Mediante la rejilla GRAI se identifica la siguiente limitación: el flujo de materiales total está limitado por el

\begin{tabular}{|c|c|c|}
\hline $\begin{array}{l}D+20: 2 \text { vs } D+13: 2 \\
D+20: 2 \text { vs } D+13: 1\end{array}$ & $\begin{array}{l}\text { CADENA SUMINISTRO } \\
\text { INTERNA }\end{array}$ & $\begin{array}{l}\text { CADENA SUMINISTRO } \\
\text { ERTERNA }\end{array}$ \\
\hline $\begin{array}{l}\text { TEenologiasue } \\
\text { INFormetín }\end{array}$ & $\begin{array}{l}\text { - Generaciòn de órdenes ae } \\
\text { fabricación en el subcontratista principal. } \\
\text { Eenergacion y emesión de pedidas de } \\
\text { aprovisionamientos a provetedores. }\end{array}$ & - Recepciôn de pedidos de proveedorę \\
\hline 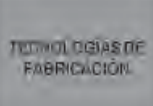 & $\begin{array}{l}\text {-Tamanio de jotas de preduccion } \\
\text { - Eficiencia duellics de botella. } \\
\text { - Trabaja en proceso }\end{array}$ & $\begin{array}{l}\text { - Tamario de lotes de procuccion en } \\
\text { provedores. }\end{array}$ \\
\hline opnarizacióy & 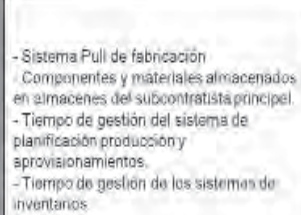 & $\begin{array}{l}\text { - Componentes y materiales almadenados } \\
\text { en aimacenes de procucto terminacio de } \\
\text { preveedores. } \\
\text { - Gisternas de suministro de proveedores } \\
\text { eficientes. }\end{array}$ \\
\hline
\end{tabular}

Fig. 10: Tecnologías de la información, tecnologías de fabricación y factores de organización críticos que se tuvieron en cuenta al implementar el Sistema Futuro horizonte de planificación y el periodo de planificación a nivel de planificación maestra de la producción. Esto tiene un importante impacto en el periodo de maduración del sistema y consecuentemente en la fecha de entrega de los stocks de componentes necesarios para trabajar sin interrupciones.

Para el diseño del futuro SPIM se establecen varias alternativas de reducción del horizonte y el periodo de la planificación maestra de producción. En concreto se analiza el sistema de planificación " $\mathrm{D}+13: 2$ " con un período de planificación de 2 días y un horizonte de planificación de 13 días. La otra alternativa es " $\mathrm{D}+13: 1$ ", con un período de planificación de 1 día y un horizonte de 13 días. En teoría, el nuevo sistema podría funcionar con menos trabajo en proceso, ya que supone una respuesta más rápida de las plantas de fabricación para el montaje final.

Sin embargo, para analizar la eficacia del futuro sistema, se monitorizan aspectos de tecnologías de información, tecnologías de fabricación y organización de la cadena de suministro interna y externa (Fig.10).

Al analizar la eficiencia para operar con el futuro SPIM, las herramientas DGRAI permiten realizar un seguimiento pudiendo comprobar si hay cuellos de botella o problemas de capacidad en la planificación de la producción y el inventario. Con el fin de comparar la calidad y el comportamiento dinámico de ambos sistemas, se lleva a cabo una simulación de un año de duración mediante DGRAI (Fig. 11). En la Fig.

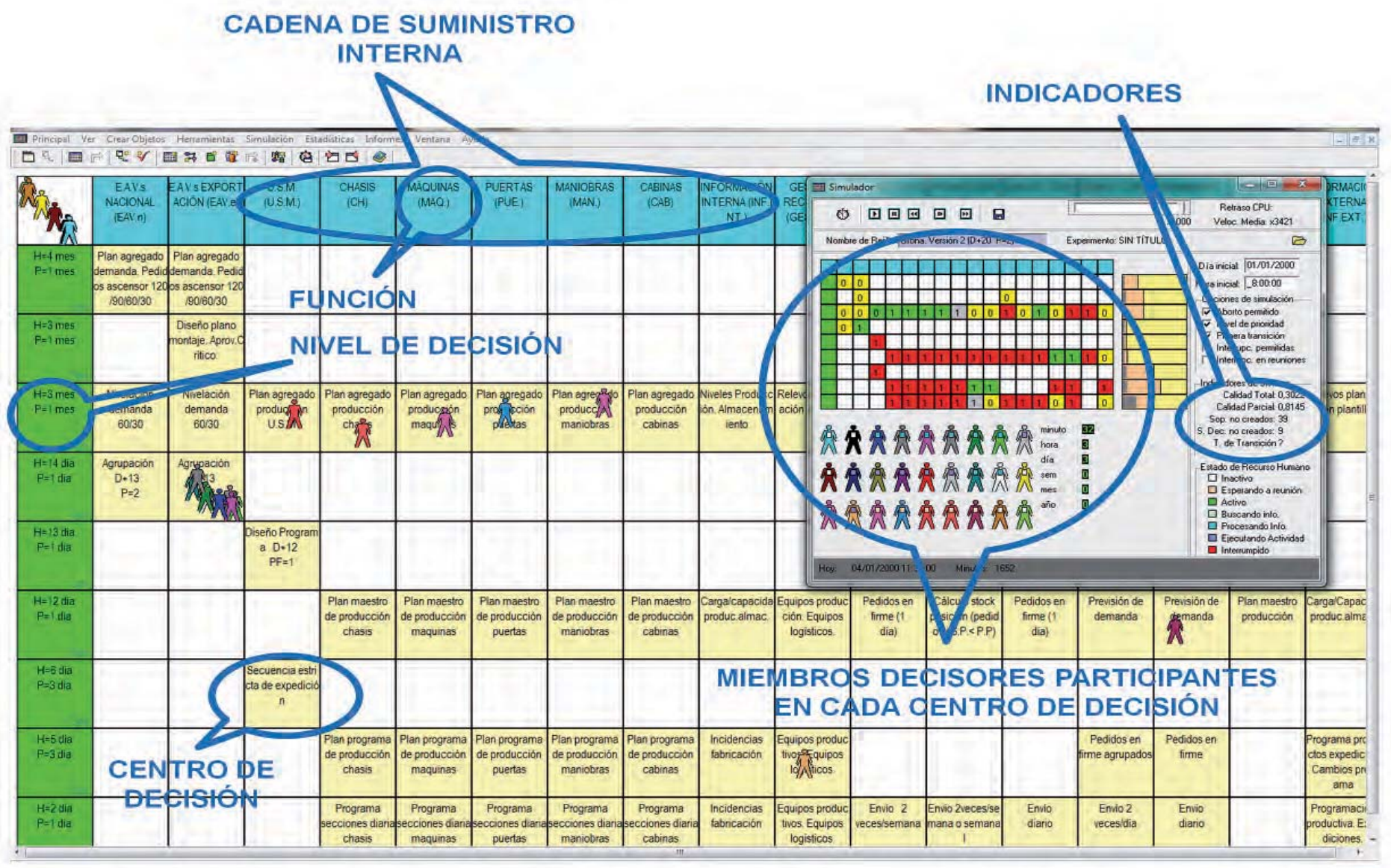

Fig. 11: Simulación en DGRAlLas conclusiones obtenidas fueron: 
11 se muestra un instante concreto de la simulación donde se pueden observar varias informaciones. Por ejemplo, la pantalla de datos que aparece a la derecha muestra qué centros de decisión han sido ya ejecutados (color amarillo), cuáles están siendo ejecutados (color verde) y cuáles están sin ejecutar (color rojo).

- Ambos sistemas de planificación alternativos son correctos desde el punto de vista de la coordinación de suministros. La simulación no evidencia problemas de sincronización entre la cadena de suministro externa e interna.

- En relación a la dedicación de recursos humanos al sistema, el sistema de planificación " $\mathrm{D}+13: 2$ " necesitaba un recalculo cada dos días del plan maestro de producción, frente al sistema de planificación " $\mathrm{D}+13: 1$ " que utiliza un recalculo diario. La simulación muestra que el " $\mathrm{D}+13: 1$ " necesitaba un 40\% más de horas de dedicación de los recursos humanos para la toma de decisiones que el " $\mathrm{D}+13: 2$ " por lo que es más caro desde el punto de vista de coste de personal.

- Con respecto al impacto de la capacidad de los recursos humanos en el rendimiento del sistema de soluciones, un indicador interesante es la cantidad de decisiones que tienen en cola los responsables de tomar decisiones a lo largo del tiempo. Ambos sistemas de planificación tienen un comportamiento similar en cuanto al número máximo de decisiones en cola, pero el tiempo de cola es mayor en "D+13:1": el número medio de decisiones en la cola es de un $50 \%$ más alto en " $D+13: 1$ " que en "D+13:2". En particular, el jefe de planificación de la cadena de suministro interna se encuentra especialmente sobrecargado en todo momento en "D+13:1".

- Referente a la evolución del indicador de la Calidad Total de Sistema de Decisión (TDQS), el cual mide de manera agregada el buen funcionamiento del sistema de decisión, el sistema " $\mathrm{D}+13: 1$ " es, en promedio, un 4\% mejor respecto al " $D+20: 2$ ". TDQS se calcula como la media ponderada de la calidad de las decisiones en un momento dado. La calidad de una decisión depende de la calidad de la información utilizada y de la calidad de los recursos humanos que la toman, y ésta disminuye con el tiempo hasta su regeneración en cada período de revisión.

Evaluando los resultados que se consiguieron simulando ambos escenarios y siguiendo el consejo de los investigadores que han llevado a cabo el estudio, la empresa finalmente ha tomado la decisión de implantar el sistema " $\mathrm{D}+13: 2$ ".

\section{RESULTADOS EN TÉRMINOS DE EFECTIVIDAD}

Los gerentes de la unidad de negocio de ascensores valoran las ventajas y desventajas expuestas a través las herramientas GRAI y deciden poner en práctica el nuevo SPIM. Después de dos años desde el comienzo del proceso de reingeniería las mejoras más destacables son:

- Una reducción de un 35\% del plazo de entrega y de un $10 \%$ de aumento en el cumplimiento pedidos de las obras de montaje.

- Una reducción de un 30\% del trabajo en proceso en el subcontratista principal.

- Un 30\% de reducción de stock en los almacenes de proveedores J.I.T. de módulos.

- Un $20 \%$ de incremento de stock en proveedores contra pedido obligados a trabajar como montaje contra pedido con un nuevo punto de desacople.

- Un $30 \%$ de reducción de stock en parte de los proveedores contra almacén.

\section{CONCLUSIONES}

El equipo multidisciplinar involucrado en el proyecto de reingeniería ha valorado como muy útil el método planteado. A su vez ha demostrado la aplicabilidad de la rejilla GRAI y la simulación DGRAI como herramientas analíticas en entornos de sistemas de producción de ingeniería bajo pedido. Además, se ha ampliado la aplicación del modelo DGRAI a cadenas de suministro con sistemas de producción de ingeniería bajo pedido, en los que la localización de los proveedores y los períodos de tránsito tienen especial importancia, integrándolo en la metodología propuesta en el presente artículo.

En relación a la integración operativa entre los proveedores y los subcontratistas principales, los beneficios de la reducción de stock se pueden conseguir en los almacenes de los proveedores/clientes o en los almacenes del propio subcontratista. Por lo tanto, el esfuerzo de realizar el análisis y su negociación es necesario en ambos casos.

Como futuras líneas de investigación los autores de este artículo sugieren las siguientes:

- Identificar las mejores prácticas de contratación que permitan una mejor colaboración entre los subcontratistas y los proveedores en sistemas de producción por proyectos que cubre una amplia gama de productos o servicios, además de los tipos de acuerdos de los niveles de capacidad de los proveedores.

- Analizar el impacto de la localización de los proveedores cuando se suministran módulos complejos en secuencia y sincronización con la producción para cumplir con los tiempos de respuesta cortos y las alternativas para desacoplar los proveedores con períodos de tránsito grandes.

- Conseguir una nivelación de los pedidos en los Sistemas de Producción de Ingeniería Bajo Pedido, ya que, una vez que los pedidos están diseñados, podrían ser retenidos para tratar de satisfacer la fecha de entrega de los diferentes clientes (pedidos de exportación, pedidos regionales...), haciendo que se nivele la capacidad de la planta productiva.

- Extender el Método GRAI y el Modelo DGRAI a otros análisis de sistemas de producción de Ingeniería bajo pedido. 


\section{BIBLIOGRAFÍA}

- Acur N and Biticci U. (2000), "Active assessment of strategy performance in proceedings of the IFP WG 5.7", International Conference on Production Management, Tromso, Norway. - Aguilar-Sommar RS, Poler R. (2006) "Integrated analysis of the production planning process using Trampolin and DGRAI as process modelling tools" Production Planning \& Control, Vol. 17, No. 1, January 2006, 31-43

- Anumba CJ, Baugh C, Khalfan MA. "Organisational structures to support concurrent engineering in construction". Industrial Management Data System. 2000 Vol.102 p.260-270

- Anumba CJ, Siemieniuch CE, Sinclair MA. "Supply chain implications of concurrent engineering" Physical Distribution and Logistic. 2000 Vol.30-7 p.566-597

- Akif JC. (1991) "Consistency analysis of P.M.S. Based on GRAI modelling. Computer Applications in Production and Engineering: Integration Aspects". Elsevier Science Publishers B.V. (Noth-Holland) - Badr Haque. "Problems in concurrent new product development: an in-depth comparative study of three companies". Integrated Manufacturing System. 2003 Vol.14-3 p.191-207.

- Browne J, Sackett PJ and Wortmann JC. "Future Manufacturing Systems-towards the extended enterprise". Computers in Industry, 1995 Vol.25 p.235-254.

- Chan Albert PC, Fan Linda CN, Yu Ann TW. "Construction process reengineering: a case study". Logistic Information Management, 1999 Vol.12-6 p.467-75.

- Caron F. and Fiore A. "Engineer to order companies : how to integrate manufacturing and innovative processes". Project Management. 1995 Vol.13-5 p.313-319.

- Childe SJ. "The extended enterprise-a concept of cooperation". Production Planning and Control. 1998. Vol. 9-4 p.320-327 - Doumeingts G. "Méthode GRAI: Méthode de conception des systémes en productique". Thése d'état : Automatique. .1984, Université de Bordeaux

- Errasti A, Poler R, Oyarbide A and Santos J. "Supply chain improvement based on GRAI Method: an empirical study" Proceedings of 13th Euroma International Conference 2006, Glasgow, Great Britain

- Errasti A, Poler R, Eguren, JA, Igartua, JI." Mejora de un Sistema de Producción por Proyectos basada en la metodología GRAl : un estudio empírico", XI Congreso de Ingeniería de Organización, 2007, Madrid.

- Errasti A, Beach R and Apaolaza U. "Close coupling value chain functions to improve subcontractor manufacturing performance" Project Management, 2008.

- Fawcett S. and Magnan G. "The rhetoric and reality of supli chain integration", Physical Distribution and Logistics Management. 2002 Vol.32-5 p.339-361

- Feurer $\mathrm{R}_{1}$ Chaharbaghi $\mathrm{K}$ and Wargin J. "Analysis of strategy formulation and implementation at Hewlet Packard", Management Decision. 1995 Vol.33-10 p.4-16.

- Gunasekaran A, Love PED. "Concurrent engineering a multidisciplinary approach for construction". Logist Information Management. 1998. Vol.11-5 p.295-300.

- Gunn TG. (1987), "Manufacturing for Competitive Advantage: Becoming a World Class Manufacturer", Ballinger Publishing Company, Boston M.A.

- Hernández JE, Mula J, Ferriols F, Poler R. (2008) "A conceptual model for the production and transport planning process: An application to the automobile sector" Computers in Industry 59 842-852 - Hoekstra S and Romme J. Integrated Logistics Structures: Deveolping Customer Oriented Goods Flow. London: Mc Graw-Hill, 1992. 180p. ISBN-10: 0831130377

- Jagdev HS and Browne J." The extended Enterprise-a context for manufacturing".Production Planning and Control. 1998 Vol.9-3 p.
216-229

- Huan Neng Chiu "The integrated logistics management system: a framework and case study" Physical Distribution \& Logistics Management, 1995 Vol. 25-6 p.4-22

- Kolltveit BJ. "The importance of the early phase: the case of construction and building project". Project Management. 2004 Vol.22-5 p.45-51.

- Mahmoud-Jouini SB, Midler C, Garel G. "Time-to-market vs. time-todelivery: managing speed in engineering, procurement and construction projects". Project Management.2004 Vol.22-5 p.59-67 - Marucheck A, Pannesi R and Anderson C. "An exploratory study of the manufacturing strategy in practice" Operations Management. 1990 Vol.9-1 p.101-23.

- Ngowi AB. "Construction procurement based on concurrent engineering principles". Logistic Information Management. 2000 Vol.13-6 p.361-8.

- Olhager J. "Strategic positioning of the order penetration point". Production Economics, 2003 Vol. 85-3 p.319-29

- Platts KW. "Manufacturing audit in the process of strategy formulation", PhD dissertation, 1990.University of Cambridge, Cambridge.

- Poler R and Lario FC. "Simulation using the Dynamic Model of Decision Systems", International Conference on industrial Engineering and Production Management (IEPM), Quebec IEEE Press, 2001 p.1004-1012.

- Poler R, Lario FC and Doumeingts G. "Dynamic Model of Decision Systems (DMDS)" Computers in Industry, 2002 Vol.49, p.175-193. - Porter ME. Competitive strategy: techniques for analyzing industries and competitors. New York: The Free Press; 1980. 397p. ISBN-10: 0684841487

- Porter ME. Competitive advantage. New York: The Free Press; 1985. 576p. ISBN 0029250900

- Rudberg M, Wikner J. (2004), "Mass customization in terms of the customer order decoupling point", Production Planning \& Control, Vol. 15 No.4, pp.445-58.

- Salvador F, Rungtusanatham M , Forza C And Trentin A..

"Understanding synergies and trade-offs between volume flexibility and mix flexibility in Build-to-Order strategies". Proceedings of 12th Euroma International Conference. 2005, Budapest, Hungary - Schermerhorn J. Management for productivity New York: Wiley; 1993. 848p. ISBN-10: 0471599913

- Slack N, Chambers S. And Johnston R. Operations Management, $4^{\mathrm{a}}$ edición. Londres : Pearson, 2004. 670p. ISBN: 978-0-273-73046-0

- Söderlund J. "On the broadening scope of the research on projects: a review and a model for analysis". Project Management. 2004 Vol.226 p.55-67.

- Towill DR and McCullen PL. "The impact of agile manufacturing on supply chain dynamics ", Logistics Management. 1999. Vol.10-1, p.83-96

- Thomas S. and Skitmore M. (2002) "Contractor Risks in Design, Novate and Construct Contracts". Project Management. 2002.

Vol.20-2 p. 119-126

- Vignalli C."The marketing management process and heuristic devices: an action research investigation". Market Intelligence Plann. 2003. Vol.21-4 p.205 - 219.

- Wadhwa S. (1998) "Simulation study of shop floor control systems under decision and information delays using GRAI macro reference model" 5th International Workshop AUGRAI. Glasgow.

- Westbrook R. "Action Research : a new paradigm for research in production and opérations management ", Operations and Production Management, 1995 Vol.15-12 p.6-20

- Yin R.K. Case Study Research: Design and Methods. $3^{\text {a }}$ edición. Londres : Sage Publications, 1994. 192p. ISBN 0-7619-1946-5 - Zhenxin Yu, Hong Yan TC,. Edwin Cheng "Benefits of information sharing with supply chain partnerships" Industrial Management and Data Systems. 2001. Vol.101-3 p.114-119 